\title{
CAPITAL BUDGETING IN ARBITRAGE-FREE MARKETS
}

\author{
By Jörg Laitenberger and Andreas Löffler*
}

\begin{abstract}
In capital budgeting problems future cash-flows are discounted using the expected one period returns of the investment. In this paper we establish a theory that relates this approach to the assumption that markets are free of arbitrage. Our goal is to uncover implicit assumptions on the set of cash-flow distributions that are suitable for the capital budgeting method. As results we obtain that the set of admissible cash-flow distributions is large in the sense that no particular structure of the evolution of the distributions is implied. We give stylized examples that demonstrate that even strong assumptions on the return distributions do not restrain the shape of the cash-flow distributions. In a subsequent analysis we characterize the cash-flow distributions under the additional assumption of a deterministic dividend yield. In this case strong properties for the evolution of the distributions can be obtained.
\end{abstract}

keywords: cost of capital, capital budgeting

JEL-class.: G 31, D46

*The authors thank the Verein zur Förderung der Zusammenarbeit von Lehre und Praxis am Finanzplatz Hannover e.V. for financial support. 


\section{INTRODUCTION}

It is well known today that in arbitrage free markets the value of a claim is given by the sum of its expected cash-flows discounted at the riskless interest rate. The expectation is taken with respect to the so-called risk neutral measure that is usually different from the subjective probability measure of the investor. In the context of project valuation both scientist and practitioners tend to use the so called net present value method, which consists in discounting the expected cash-flows with the periodby-period cost of capital. The expectation is now taken with regards to the subjective probability of the investor. The cost of capital are given by the expected rate of return of the investment. In this paper we establish a theory that relates the latter approach to the assumption that markets are free of arbitrage. Our goal is to uncover implicit assumptions on the set of cash-flow distribution that are suitable for the capital budgeting method.

If the discount rate is derived from an equilibrium model as the CAPM the above problem reduces to the question under what assumptions a myopic valuation principle can be applied. This problem was considered by Fama (1977), Sick (1986), Black (1988) and Franke (1984).

Fama (1977) investigated the case of a single cash-flow realization in some future period. Later Fama (1996) has pointed out that in this case the distribution of cash-flows tend to become more and more skewed right in later periods when the distribution of one-period single returns are roughly symmetric. Sick (1986) investigated comparable additive or multiplicative cash-flow process (his assumptions A2 and A3). In Black (1988) both the cash-flows of the project and the cash-flow of the market portfolio are joint normal.

Whereas Fama (1977), Sick (1986) and Black (1988) considered cashflows having a particular stochastic structure, Franke (1984) instead made no assumptions for the (exogenous) dividend process except regularity conditions. Using a multiperiod exchange economy with HARA investors he derived conditions for a period-by-period application of one-period 
asset pricing models. Since we will not use an equilibrium model our paper does not directly compare to these results.

Within an arbitrage model, Richter (2001) tackled the problem of constant discount rates. He used a binomial model and was able to derive equations that implied a constant discount rate for future cash-flows. Therefore, a particular stochastic structure of the cash-flows is evident. In particular, within the binomial model only one ratio of the growth rate for up- and down-movements will lead to a constant discount rate.

In our short note we relate the question of constant cost of capital not to an equilibrium concept. Furthermore, we do not want to restrict ourselves to a particular distribution of future cash-flows. Instead, we will use a fairly general model to derive our results. We start with a definition of discount rates as future returns and ask under what assumptions these discount rates can be used in capital budgeting and are in particular constant. It turns out that the set of admissible cash-flow distributions is large in the sense that no particular structure can be found.

A first example shows that even in a model where cash-flows posses any given structure in every future time period, cost of capital can be constant. Therefore, maybe counterintuitively, the variance or the skewness of the cash-flow distributions do not change through time. If we add another assumption about the dividend yield of the firm it will turn out that the cash-flow increments have to be uncorrelated. This is much weaker than saying that the increments are independent as it is usually assumed in the random walk hypothesis 11 These conditions are not only sufficient but in a particular sense also necessary for the cost of capital to be constant.

The next section presents an example of a firm where the cash-flows have an arbitrary stochastic structure in any future time but cost of capital are constant and the market is free of arbitrage. A theory of cost of capital used in capital budgeting is given in the following section. The last section closes the paper.

\footnotetext{
${ }^{1}$ Uncorrelated variables are necessarily independent only if they are joint normal.
} 


\section{Constant Cost of CAPITAL: An EXAMPle}

Let $(\Omega, \mathcal{F}, P)$ be a probability space and a filtration $\mathcal{F}_{t}$ (for details see Williams (1991)). The world ends in $T, T=\infty$ is possible. A firm realizes uncertain cash-flows $\tilde{C} F_{t}$ that are $\mathcal{F}_{t}$-measurable. The value of the firm at time $t$ is $\tilde{V}_{t}$. The riskless interest rate is for simplicity time independent and $r_{f}$. If the market is free of arbitrage there is a risk neutral probability measure $Q$ such that the following is true (for a proof see for example Back \& Pliska (1991))

(1) $\tilde{V}_{t-1}=\frac{\mathrm{E}_{Q}\left[\tilde{V}_{t}+\widetilde{C} F_{t} \mid \mathcal{F}_{t-1}\right]}{1+r_{f}}$,

where $\mathrm{E}_{Q}\left[\cdot \mid \mathcal{F}_{t}\right]$ is the conditional expectation under the risk neutral probability. Our purpose is to clarify the relationship between arbitrage free markets and capital budgeting. To this end we now define the rate of return: At any future time $t$ the rate of return one period ahead from holding a share of the company is

(2) $\tilde{r}_{t}:=\frac{\tilde{V}_{t}+\tilde{C} F_{t}}{\tilde{V}_{t-1}}-1$.

In a world with uncertainty $\tilde{r}_{t}$ will be a random variable. The expectation of the rate of return $\tilde{r}_{t}$ with respect to the information $\mathcal{F}_{t-1}$ will be denoted as the cost of capital of the investment in period $t$. Therefore, the cost of capital are the conditional expectations of the one period returns:

$$
\tilde{k}_{t}=\mathrm{E}\left[\tilde{r}_{t} \mid \mathcal{F}_{t-1}\right]
$$

Let us now turn to the capital budgeting problem. From (2) it immediately follows that

$$
\tilde{V}_{t-1}=\frac{\mathrm{E}\left[\tilde{V}_{t}+\tilde{C} F_{t} \mid \mathcal{F}_{t-1}\right]}{1+\widetilde{k}_{t}} .
$$

But for capital budgeting it is necessary to assume that the cost of capital are deterministic. Only in this case (2) implies

$$
\tilde{V}_{t-1}=\frac{\mathrm{E}\left[\tilde{C} F_{t} \mid \mathcal{F}_{t-1}\right]}{1+k_{t}}+\frac{\mathrm{E}\left[\tilde{V}_{t+1}+\tilde{C} F_{t+1} \mid \mathcal{F}_{t-1}\right]}{\left(1+k_{t+1}\right)\left(1+k_{t}\right)}
$$


Notice that for deterministic cost of capital the rate of returns will be necessarily serially uncorrelated. For $s>t$ :

$$
\begin{aligned}
\operatorname{Cov}\left[\tilde{r}_{s}, \tilde{r}_{t}\right] & =\mathrm{E}\left[\tilde{r}_{s} \tilde{r}_{t}\right]-\mathrm{E}\left[\tilde{r}_{s}\right] \mathrm{E}\left[\tilde{r}_{t}\right]=\mathrm{E}\left[\tilde{r}_{t} \mathrm{E}\left[\tilde{r}_{s} \mid \mathcal{F}_{t}\right]\right]-k_{s} k_{t} \\
& =\mathrm{E}\left[\tilde{r}_{t} k_{s}\right]-k_{s} k_{t}=0 .
\end{aligned}
$$

This is a priori not restraining much the distribution of $\tilde{C} F_{t}$ and $\tilde{V}_{t}$. Indeed, since the one period returns divide between dividends $\left(\widetilde{C} F_{t}\right)$ and capital gains $\left(\tilde{V}_{t}-\tilde{V}_{t-1}\right)$ almost any sequence of future cash-flows or alternatively any sequence of future firm values can be constructed that imply rate of returns as described by (2). If market conditions allow for estimating the one period returns according to the CAPM or the ICAPM, this restricts the set of acceptable return distributions $\tilde{r}_{t}$, but does not change anything to the indeterminacy of the cash-flow distribution as we will show in an example below.

To this end consider a sequence of iid random variables $\tilde{C} F_{t}$ for all $t$. Let two probability measures $Q, P$ given such that

$$
\mathrm{E}_{Q}\left[\widetilde{C} F_{t}\right]=r_{f}, \quad \mathrm{E}\left[\widetilde{C} F_{t}\right]=k \neq r_{f}
$$

The filtration $\mathcal{F}_{t}$ is implicitly determined by the sequence of random variables $\widetilde{C} F_{1}, \ldots, \widetilde{C} F_{t}$. The value of the firm is equal to one

$$
\tilde{V}_{t}:=1
$$

Notice that the value of the firm is not a random variable anymore, although we continue to use the tilde. It is straightforward to verify that this model is free of arbitrage: given the definition of the riskless interest rate we have

$$
\frac{\mathrm{E}_{Q}\left[\tilde{C} F_{t}+\tilde{V}_{t} \mid \mathcal{F}_{t-1}\right]}{\tilde{V}_{t-1}}=1+r_{f} .
$$

showing our model is free of arbitrage. On the other hand, the cost of capital are given by

$$
\frac{\mathrm{E}\left[\tilde{C} F_{t}+\tilde{V}_{t} \mid \mathcal{F}_{t-1}\right]}{\tilde{V}_{t-1}}=1+k .
$$


We arrive at a situation where the cost of capital of our firm are constant although the risk of the cash flows does not change: in every period the cash flows are given by the same random variable. The risk of the cash-flows does not increase. The value of the firm can be evaluated by discounting the expected cash-flows using the cost of capital. In this case the value of the firm obtains as

$$
V_{0}=\sum_{t=1}^{\infty} \frac{\mathrm{E}\left[\tilde{C} F_{t}\right]}{(1+k)^{t}}=1
$$

Notice that in our example the expectation and even the variance of the future cash-flows remain constant. Hence, constant cost of capital do not imply a greater risk measured in terms of variance. This effect is only obtained when the cash-flows under consideration are correlated. In our example this is the case. A straightforward calculation shows that any two cash-flow increments are highly correlated ${ }^{2}$

In this sense the behavior of cash-flows and returns observed in our example is only obtained under the very special conditions the example was built on. In a real world environment this seems to be a rather unusual behavior since the value of the firm is not a random variable anymore. In the following we are interested in the behavior of cash-flows when we prevent this sort of correlation. In this case it turns out that much more can be said about the implications of constant or deterministic cost of capital.

\section{CASH-FLOWS WITH A DETERMINISTIC DIVIDEND YIELD}

Define the dividend ratio of a cash-flow distribution as

(4) $\tilde{d}_{t}:=\frac{\tilde{C} F_{t}}{\tilde{V}_{t}}$

\footnotetext{
${ }^{2}$ When $\tilde{C} F_{t}$ are independent, then obviously the increments $\tilde{C} F_{t}-\tilde{C} F_{t-1}$ are correlated with

$\operatorname{Cov}\left[\tilde{C} F_{t}-\tilde{C} F_{t-1}, \tilde{C} F_{t+1}-\tilde{C} F_{t}\right]=-\operatorname{Var}\left[\tilde{C} F_{t}\right]$.
} 
It is a standard assumption in multiperiod valuation problems to assume that this ratio is deterministic 3 If both the dividend ratio and the cost of capital are deterministic then the cash-flows have to satisfy the relation

$$
\forall t \quad \mathrm{E}\left[\tilde{C} F_{t+1}-\tilde{C} F_{t} \mid \mathcal{F}_{t}\right]=g_{t+1} \cdot \tilde{C} F_{t} .
$$

where $g_{t}$ is deterministic. Before proving our main result we want to discuss the above property of future cash-flows. We claim that our assumption is equivalent to saying that the increase in future cash-flows are (conditional) uncorrelated. This is much weaker than saying (as it is usually assumed in the random walk hypothesis) that the increments are independent from past cash-flows. Only for normally distributed random variables uncorrelated variables are necessarily independent.

PROPOSITION 1. For condition (5) to hold it is sufficient and necessary that the cash-flows can be written as

$$
\widetilde{C} F_{t+1}=\left(1+g_{t+1}\right) \tilde{C} F_{t}+\tilde{\varepsilon}_{t+1}
$$

where $\varepsilon_{t}$ are uncorrelated with expectation zero.

Proof. Let $\tilde{\varepsilon}_{t}$ be defined as in (6). Using (5) these $\varepsilon_{t}$ obviously have expectation zero. The correlation between two increments can be written as

$$
\operatorname{Cov}\left(\tilde{\varepsilon}_{t+1}, \tilde{\varepsilon}_{s+1}\right)=\mathrm{E}\left[\left(\widetilde{C} F_{t+1}-\left(1+g_{t+1}\right) \widetilde{C} F_{t}\right) \cdot\left(\tilde{C} F_{S+1}-\left(1+g_{s+1}\right) \widetilde{C} F_{s}\right)\right]
$$

Let $s<t$, then using the law of iterated expectation (see for example Williams (1991, p. 88)) this can be rearranged to

$$
\operatorname{Cov}\left(\tilde{\varepsilon}_{t+1}, \tilde{\varepsilon}_{s+1}\right)=\mathrm{E}\left[\left(\tilde{C} F_{s+1}-\left(1+g_{s+1}\right) \widetilde{C} F_{s}\right) \cdot \mathrm{E}\left[\widetilde{C} F_{t+1}-\left(1+g_{t+1}\right) \widetilde{C} F_{t} \mid \mathcal{F}_{s+1}\right]\right]
$$

But the right hand side is zero since

$$
\mathrm{E}\left[\left(\tilde{C} F_{t+1}-\left(1+g_{t+1}\right) \tilde{C} F_{t}\right) \mid \mathcal{F}_{s+1}\right]=\mathrm{E}\left[\mathrm{E}\left[\left(\tilde{C} F_{t+1}-\left(1+g_{t+1}\right) \tilde{C} F_{t}\right) \mid \mathcal{F}_{t}\right] \mid \mathcal{F}_{s+1}\right]=0
$$

\footnotetext{
${ }^{3}$ This is for instance the underlying assumption in Merton's proportional dividendyield option pricing model, see Merton (1974). Geske is more general and used an independent dividend yield in his model, see Geske (1978).
} 
and hence one part of our claim is shown. The other direction is trivial.

We furthermore notice that the claimed structure of future cash-flows (5) seems to be the discrete time analog of the assumption of a Brownian Motion. In the later case the stock price process satisfies

$$
\frac{d S}{S}=(r+d) d t+\sigma d W
$$

and this is the same as to say that the (infinitesimal) increase $d S$ is uncorrelated to the current stock prize.

At first glance it is not clear what rôle the assumption of a deterministic dividend ratio plays. Therefore we will show a much stronger result. We will prove that this deterministic dividend yield is an undispensible condition. We show in the context of capital budgeting that deterministic cost of capital and our cash-flow assumption imply a deterministic dividend yield. Furthermore, it will turn out that our cash-flow property is not only necessary for cost of capital to be constant but also sufficient. Our result is summarized in the following proposition.

Proposition 2. Assume the market is free of arbitrage. If two of the following conditions are satisfied the third follows

(i) the cost of capital $\tilde{k}_{t}$ are deterministic,

(ii) there are real numbers $g_{t}>-1$ such that the relation (5) holds,

(iii) the dividend ratios $\tilde{d}_{t}$ are deterministic with $d_{t}>0$.

Proof. We start with $(i),(i i) \Longrightarrow$ (iii). From (3), (5) and the law of iterated expectation it follows for all $t$

$$
\tilde{V}_{t}=\tilde{C} F_{t} \cdot \sum_{s=t+1}^{T} \frac{\left(1+g_{t+1}\right) \cdots\left(1+g_{s}\right)}{\left(1+k_{t+1}\right) \cdots\left(1+k_{s}\right)}=: \tilde{C} F_{t} \cdot d_{t}^{-1}
$$

hence, the dividend ratios $d_{t}$ is deterministic. 
Now (ii), (iii) $\Longrightarrow($ i). We have from (1), (3) and

$$
1+\tilde{k}_{t}=\frac{\mathrm{E}\left[\left(1+d_{t}^{-1}\right) \widetilde{C} F_{t} \mid \mathcal{F}_{t-1}\right]}{d_{t-1}^{-1} \widetilde{C} F_{t-1}}=\left(1+d_{t}^{-1}\right)\left(1+g_{t}\right) d_{t-1}
$$

and hence the cost of capital must be deterministic.

To show $(i),($ iii $) \Longrightarrow($ ii) we start with

$$
\begin{aligned}
\left(1+k_{t}\right) V_{t-1} & =\mathrm{E}\left[\tilde{C} F_{t}+\tilde{V}_{t} \mid \mathcal{F}_{t-1}\right] \\
\frac{1+k_{t}}{1+d_{t}^{-1}} d_{t-1} \tilde{C} F_{t-1} & =\mathrm{E}\left[\tilde{C} F_{t} \mid \mathcal{F}_{t-1}\right]
\end{aligned}
$$

and this is indeed (5).

In our present formulation we assume that the distributions of cashflows satisfy $\widetilde{C} F_{t} \neq 0$ in every period $t \leq T$. This rules out for instance the case of distributions having a single cash-flow $\widetilde{C} F_{T}$ at time $T$ and no cash-flows at any other time. In this case condition (5) would enforce $\mathrm{E}\left[\tilde{C} F_{T}\right]=0$. In order to allow for zero cash-flows at some periods, $\tilde{C} F_{t}$ can be replaced by $\tilde{V}_{t}$ in 5 leaving all results of the proposition valid.

Note that under the assumptions of proposition 3 the expected returns $k_{t}$ are not only the appropriate cost of capital for the entire firm, but every single cash-flow $\tilde{C} F_{t}$ is to be valued using $k_{t}$ as discount factors. This obtains as follows. Capital budgeting in arbitrage free markets establishes the following equation for $\tilde{V}_{u}$

$$
\tilde{V}_{u}=\sum_{t=u+1}^{T} \frac{\mathrm{E}_{Q}\left[\tilde{C} F_{t} \mid \mathcal{F}_{u}\right]}{\left(1+r_{f}\right)^{t-u}}=\sum_{t=u+1}^{T} \frac{\mathrm{E}\left[\tilde{C} F_{t} \mid \mathcal{F}_{u}\right]}{\prod_{s=u+1}^{t}\left(1+k_{s}\right)} .
$$

We now demonstrate that not only both sums lead to the same result but that this is also true for every single entry. Hence, cost of capital turns out to be a simple way of evaluating the expected cash-flows under the subjective probability measure. Cost of capital are not only expected returns but also appropriate discount factors:

PROPOSITION 3. If the conditions of proposition 3 are satisfied for any $t>s \geq 1$, then the value of a single cash-flow $\widetilde{C} F_{t}$ at time $s$ obtains as

(7) $\frac{\mathrm{E}_{Q}\left[\tilde{C} F_{t} \mid \mathcal{F}_{s}\right]}{\left(1+r_{f}\right)^{t-s}}=\frac{\mathrm{E}\left[\tilde{C} F_{t} \mid \mathcal{F}_{s}\right]}{\prod_{u=s+1}^{t}\left(1+k_{u}\right)}$. 
Proof. We show the claim for $s=t-1$. Since the dividend ratio is deterministic we have

$$
\begin{aligned}
\frac{\mathrm{E}_{Q}\left[d_{t}^{-1} \tilde{C} F_{t}+\tilde{C} F_{t} \mid \mathcal{F}_{t-1}\right]}{1+r_{f}} & =\tilde{V}_{t-1}=\frac{\mathrm{E}\left[d_{t}^{-1} \tilde{C} F_{t}+\tilde{C} F_{t} \mid \mathcal{F}_{t-1}\right]}{1+k_{t}} \\
\Longrightarrow \quad \frac{\mathrm{E}_{Q}\left[\tilde{C} F_{t} \mid \mathcal{F}_{t-1}\right]}{1+r_{f}} & =\frac{\mathrm{E}\left[\tilde{C} F_{t} \mid \mathcal{F}_{t-1}\right]}{1+k_{t}} .
\end{aligned}
$$

which is the claim for $s=t-1$.

By taking the expectation $\mathrm{E}\left[\cdot \mid \mathcal{F}_{t-2}\right]$ and using (5) the equation $(8)$ can be further modified to

$$
\frac{\mathrm{E}_{Q}\left[\tilde{C} F_{t} \mid \mathcal{F}_{t-2}\right]}{1+r_{f}}=\frac{\mathrm{E}_{Q}\left[\left(1+g_{t}\right) \tilde{C} F_{t-1} \mid \mathcal{F}_{t-2}\right]}{1+k_{t}}
$$

Using (8) (with $t-1$ instead of $t$ ) the right hand side simplifies to

$$
\frac{\mathrm{E}_{Q}\left[\tilde{C} F_{t} \mid \mathcal{F}_{t-2}\right]}{1+r_{f}}=\frac{\left(1+g_{t}\right) \frac{\left(1+r_{f}\right) \mathrm{E}\left[\tilde{C} F_{t-1} \mid \mathcal{F}_{t-2}\right]}{1+k_{t-1}}}{1+k_{t}}
$$

or with (5)

$$
\frac{\mathrm{E}_{Q}\left[\tilde{C} F_{t} \mid \mathcal{F}_{t-2}\right]}{\left(1+r_{f}\right)^{2}}=\frac{\mathrm{E}\left[\tilde{C} F_{t} \mid \mathcal{F}_{t-2}\right]}{\left(1+k_{t}\right)\left(1+k_{t-1}\right)} .
$$

This is the claim for $s=t-2$. Continuing our calculations we arrive at the desired result.

Another consequence of our assumptions concerns the shape of the distribution of future cash-flows. Projecting forward from time $s<t$, $\widetilde{C} F_{t}$ is given as

(9) $\tilde{C} F_{t}=\tilde{C} F_{S}\left(1+\tilde{r}_{s+1}\right) \cdots\left(1+\tilde{r}_{t}\right) \frac{d_{s}^{-1} \cdots d_{t-1}^{-1}}{\left(1+d_{s+1}^{-1}\right) \cdots\left(1+d_{t}^{-1}\right)}$.

Therefore the shape of the cash-flow distributions further ahead is principally given by the product of the one period return distributions. Obviously with such a multiplicative structure certain regularities of the 
return distributions will lead to strong properties of the cash-flow distributions. For instance, if the distribution of the returns are identical with a positive variance, then the projected distribution of the cash-flows will have increasing variances through time. This is a result that has been noticed by Fama (1996) in the case of a single cash-flow realization in the last period. In applied work many other interesting properties of the cash-flow distributions can be deduced from the return distribution and vice versa with equation (9).

\section{CONCLUSION}

Capital budgeting of future uncertain cash-flows with risk adjusted discount rates implies deterministic cost of capital. Beside, no further restriction on the shape or the evolution of the distribution of the cashflows is required when projects with many cash-flow realizations are considered. Therefore our intuition that cash-flows further ahead in the future should be more uncertain is wrong in general. Only when additional assumptions either on the dividend yield of the investment or on the cash-flow increments are made further properties of the cash-flow evolution can be deduced.

Therefore we believe that further progress in the understanding of capital budgeting problems will not arise in focussing the research on the behavior of the investment returns alone as has been done in the past but rather along meaningful assumptions on the relationship of the cash-flows themselves. A functional relationship between returns and cash-flows is obtained only when certain regularities of the evolution of the cash-flows are given, which might be the case in many applied problems.

\section{REFERENCES}

BLACK, F. (1988): “A simple discounting rule”, Financial Management, 17(2), 7-11. 
BACK, K. AND PlisKA, S.R. (1991): "On the fundamental theorem of asset pricing with an infinite state space”, Journal of Mathematical Economics, 20, 1-18.

FAMA, E. (1977): "Risk-adjusted discount rates and capital budgeting under uncertainty", Journal of Financial Economics, 5, 3-24.

FAMA, E. (1996): “Discounting under uncertainty”, Journal of Business, 69 (4), 415-428.

FRANKE, G. (1984): “Conditions for myopic valuation and serial independence of the market excess return in discrete time models", Journal of Finance, 39 (2), 425-442.

GESKE, R. (1978): "The pricing of options with stochastic dividend yield”, Journal of Finance, 33, 617-625.

MERTON, R. (1974): "On the pricing of corporate debt: the risk structure of interest rates", Journal of Finance, 29, 449-470.

RichTER, F. (2001): “Simplified Discounting Rules In Binomial Models”, Schmalenbach Business Review, 53, 175-1967.

SICK, G. A. (1986): “A certainty-equivalent approach to capital budgeting”, Financial Management, 15, 23-32.

Williams, D. (1991): Probability with Martingales. Cambridge: Cambridge University Press.

Fachbereich Wirtschaftswissenschaften, Universität Hannover,

Königsworther Platz 1, D-30167 Hannover, Germany;

JL@wacc.de and AL@wacc.de;

http://www.wiwi.uni-hannover.de/finanzierung/ 